Página inicial: 393 - Página final: 406

Tipo de artículo: de investigación

\title{
La importancia de los factores productivos y su impacto en las organizaciones agrícolas en león Guanajuato México.
}

\author{
The importance of production factors and their impact on \\ the agricultural organizations in León, Guanajuato, Mexico. \\ Recibido: noviembre 2015 Revisado: abril $2016 \quad$ Aceptado: mayo 2016 \\ Por: Fernando Saúl Infante Franco ${ }^{1}$.
}

\section{Resumen.}

Se describe y descubre el nivel de importancia que los agricultores de León, Guanajuato, México atribuyen a los factores productivos capacitación, producción, costos, apoyos gubernamentales y tecnología, relacionados con la actividad de la producción agrícola en sus parcelas. Es decir, se conoce la realidad de los agricultores para que posteriormente el gobierno, las instituciones de educación y asociaciones agrícolas, colaboren entre sí de manera coordinada en beneficio de dicha actividad agricola.

\section{Palabras claves.}

Producción agrícola, Factores productivos agrícolas, Bombeo solar de agua para riego.

\begin{abstract}
.
The level of importance of the farmers in Leon, Guanajuato, Mexico is discovered and described, which is attributed to production factors, training, production, costs, government support, and technology, related to the activity of agricultural production in their plots. That is to say, the reality of the farmers is known so that later, the government, educational institutions, and agricultural associations, cooperate with each other in a coordinated manner for the benefit of the agricultural activity.
\end{abstract}

\section{Key words.}

Agricultural Production, Productive Agricultural Factors, Solar Pump for Irrigation Water.

\footnotetext{
${ }^{1}$ Fernando Saúl Infante Franco. Doctor en Administración. Docente en Universidad Franciscana Mexicana, Universidad Iberoamericana,Escuela de Estudios Superiores de la UNAM en León, Guanajuato, México, Contacto: mantraingenieria@hotmail.com y mantrainfante@hotmail.com
} 


\section{Introducción.}

La actividad económica de una organización se puede analizar y estudiar de dos maneras, la primera es bajo el enfoque macroeconómico registrando ciertos indicadores que miden el comportamiento de los tres problemas básicos de la macroeconomía y que sirven de referencia para la elaboración de los distintos modelos que estudian estos fenómenos económicos y que son la inflación, la tasa de desempleo y la tasa de crecimiento económico. La segunda manera de estudiar la actividad económica es mediante el enfoque microeconómico que registra primordialmente la variación del producto interno bruto para establecer comparaciones con otras economías, este indicador se puede determinar a través de la oferta, la renta y la demanda.

La actividad agrícola también se puede analizar y medir tomando como referencia los tres principios de la producción -maximizar la producción y minimizar los costos, la máxima eficacia económica y la máxima ganancia- recurriendo a indicadores que midan la productividad, la rentabilidad y la eficiencia entre otros. El estudio de la producción agrícola y sus factores productivos no es un tema nuevo ya que se han realizado innumerables investigaciones de todo tipo, sin embargo, el principal aporte de este trabajo consiste en conocer y describir el nivel de importancia que los agricultores del sur de León Guanajuato atribuyen a los factores productivos y que podrian ser comparados con los que tienen organizaciones gubernamentales, educativas, de investigación y otras con el fin de analizar si los objetivos de todas las organizaciones involucradas están alineados en los objetivos de corto, mediano y largo plazo respecto a la actividad agrícola.

Es indudable que el sector agrícola enfrenta un panorama complejo pues además de cubrir la condición de la autosuficiencia alimentaria con márgenes atractivos de rentabilidad, productividad y eficiencia, deben afrontar los retos de un mundo globalizado y libre comercio que obliga aún más a las organizaciones agrícolas para reinventarse en un nuevo tipo de agricultura-social-ecológica-tecnológica, agregando de una manera más intensa y dinámica el factor tecnológico y el factor conocimiento a los tradicionales factores productivos tierra, trabajo y capital. Se podría asumir a priori que el sector agrícola requiere urgentemente de cambios sustanciales para revertir su estado actual, en donde dichos cambios podrían generarse con el factor tecnológico relacionado con el uso de sistemas fotovoltaicos de bombeo de agua para el riego agrícola.

Razon de la investigación. La idea de estudiar la actividad agrícola nace por el contacto existente desde la década de los 80's con los agricultores de la zona sur del municipio de León para el desarrollo de proyectos, asimismo, la inquietud por averiguar, conocer y describir la forma como los agricultores perciben su propia realidad y actividad agrícola en función de los factores productivos tierra, trabajo, capital, tecnología, conocimiento, y otros. Se considera a priori que de los factores productivos de la producción agrícola tierra, capital, mano de obra, tecnología, conocimiento y otros, dos de ellos pudieran ser relevantes, por una parte, el factor tecnológico relacionado con el uso de los sistemas fotovoltaicos para el bombeo de agua y por la otra los apoyos gubernamentales, donde la combinación de ambos factores podrían marcar la diferencia para que la agricultura repuntara en un panorama de competitividad y globalización.

Justificación. La enorme importancia que tiene la actividad agrícola justifica con creces el tiempo dedicado a este trabajo para conocer y estudiar la agricultura desde la perspectiva del grado de interés o importancia que los propios agricultores atribuyen a los factores productivos. 
Delimitación. Este trabajo de investigación está delimitado a los agricultores de la zona sur del municipio de León, Guanajuato en México que tengan una parcela propia y además cuenten con pozo de agua particular. Se excluye a las organizaciones agrícolas cuya producción agrícola se considera de temporal pues al depender de las lluvias, el riego agrícola empleando cualquier tipo de tecnología carece de importancia.

Contexto o marco teórico conceptual. El referente teórico lo conforman el modelo económico que rige en nuestro país, la teoría de la producción y sus principios, el proceso de producción agrícola y sus factores productivos. Se asume que en México prevalece un modelo económico caracterizado por una economía de libre mercado -enfoque neoclásicobajo la premisa del laiseez-faire, término que sintetiza la idea de dejar que los mercados funcionen en base a la oferta y la demanda y se ajusten libremente para lograr el crecimiento económico. Sin embargo, no se puede dejar fuera la intervención del gobierno -enfoque keynesiano- para que prevalezca un marco jurídico que brinde a las organizaciones una seguridad para desarrollar sus actividades en las mejores condiciones y poder así competir en los mercados globalizados.

La actividad agrícola opera dentro de este modelo económico y además bajo la influencia del fenómeno de la globalización y la firma de tratados de libre comercio con varios países. El estudio de la teoría de la producción se hace en función de los tres principios básicos; a) el principio de la "ley de los rendimientos decrecientes", b) el "principio de eficacia económica", y c) el "principio de escasez", éste último no se considera crítico en este trabajo porque, aunque la tierra es finita, es suficiente para tener niveles de producción aceptables y por lo tanto no se toma en cuenta.

De esta manera, el estudio de la producción agrícola cuando se hace bajo el principio de la ley de los rendimientos decrecientes también conocida como Ley de proporciones variables, el agricultor determina el crecimiento de su producción cuando emplea cantidades variables de un factor y cantidades fijas de los demás factores, por ejemplo, si se paga más por concepto de energía eléctrica -factor variable-manteniendo fijos los costos de diésel, gasolina y refacciones de maquinaria, entonces la producción aumentaria a un ritmo acelerado en una primera fase, más lento en una segunda hasta llegar a un punto de máxima producción, y de ahí en adelante la producción disminuirá.

En la producción agrícola se asume que hay factores fijos -tierra, capital, trabajo-; factores variables -energía eléctrica, diésel, refacciones, semillas, fertilizantes- y otros factores -tecnología, apoyos gubernamentales, conocimiento-, que en su conjunto influyen y determinan el valor de la producción. Ahora bien, cuando el estudio de la producción agrícola se hace bajo el principio de eficacia económica, el agricultor busca la mejor combinación de los factores productivos para conseguir el máximo de 'eficacia económica' representada por el menor costo posible por unidad cuando el agricultor varía uno de los factores mientras mantiene fijos los demás. Este costo minimo se logra cuando el agricultor sólo está interesado en producir una cantidad fija del producto con la combinación más eficaz de los factores productivos, asimismo, el estudio de los costos se hace clasificando los insumos en fijos y variables.

- En el corto plazo significaría que si un agricultor desea aumentar su producción agrícola deberá contratar más trabajadores -factor variable- conservando las mismas instalaciones y equipos existentes -factores fijos-, pero si desea disminuir la producción, entonces deberá despedir trabajadores sin tener que vender de inmediato edificios o maquinaria. 
- Pero en el largo plazo, donde todos los insumos son considerados variables, el agricultor logra la máxima eficacia económica cuando combina eficientemente los factores planta física, maquinaria, materia prima, mano de obra, energía eléctrica, diesel, refacciones de maquinaria y equipo dentro de los límites de su capacidad productiva. Si el agricultor busca la máxima ganancia, entonces dependerá de la relación entre los costos de producción y el ingreso total alcanzado donde el precio de venta es quien determina los ingresos, es decir, los costos y los ingresos determinan el nivel de producción de máxima ganancia.

En términos generales el agricultor realiza sus funciones de producción buscando tres objetivos; a) maximizar la producción minimizando los costos -principio de rendimientos decrecientes-; b) la máxima eficacia económica cuando combina de manera eficaz sus factores fijos y variables en el corto y largo plazo; y c) la máxima ganancia cuando la razón ingresos/costos es positiva y grande.

El proceso de producción agrícola en términos generales es el mismo sin importar que tipo de producto se cultive y es de esperarse que los costos para cada una de las actividades del proceso de producción agrícola varien dependiendo del producto -maíz, papa, frijol, trigo, alfalfa, sorgo-; insumos -semillas, fertilizantes, insecticidas- y otros -energía eléctrica, agua, diésel, refacciones-. El proceso de producción agrícola, independientemente del tamaño de la parcela, tipo de producto, tecnología empleada, consta de las actividades, preparación de la tierra, fertilización, siembra, riego, control de plaga, cosecha, almacenamiento y distribución y otros. La actividad que interesa a este trabajo es la del riego agrícola, pero no la que se refiere al uso y empleo de sistemas y técnicas de riego, sino al factor tecnológico relacionado con el uso de sistemas fotovoltaicos de bombeo de agua para el riego agrícola. Se da por hecho que el agricultor que cuenta con un pozo de agua particular, tiene la infraestructura necesaria y los recursos económicos para extraer el agua y emplearla en la actividad del riego.

\section{Metodología.}

Se consideran las perspectivas de los dos paradigmas fundamentales de investigación considerados opuestos o antagónicos, el primero es el cuantitativo que descansa en la realidad objetiva e independiente de las creencias que se tengan sobre ella pudiendo generalizar resultados, este proceso es considerado impersonal, frio, reduccionista, limitativo, cerrado y rígido; el segundo paradigma es el cualitativo que descansa en la realidad subjetiva conformado por las creencias, presuposiciones y experiencias de las personas, pudiendo abordar el problema empleando una variedad de concepciones o marcos de interpretación, este proceso es considerado vago, subjetivo, especulativo, sin posibilidad de réplica y sin datos sólidos que apoyen las conclusiones.

La combinación de ambos enfoques de investigación permitió aprovechar del enfoque cuantitativo la secuencia probatoria de sus etapas y del enfoque cualitativo su proceso en espiral donde las etapas interactúan entre sí sin seguir una secuencia rigurosa. Dada la naturaleza del problema se optó por una investigación mixta o híbrida para conocer, explorar y describir el problema planteado. Desde el enfoque cualitativo se adopta la metodologia de la teoria fundamentada para conocer y describir la forma como los agricultores perciben desde su propia realidad la actividad agrícola y sus factores productivos en función de sus usos, costumbres y tradiciones. Desde el enfoque cuantitativo se estudia el problema recurriendo a las herramientas estadísticas necesarias para analizar los datos y verificar las hipótesis planteadas. Esta estrategia metodológica permitió cubrir los objetivos planteados en torno al problema de investigación y responder a las preguntas principales de investigación que cubrieron las variables de interés. 
La estrategia metodológica adoptada en primer lugar define la investigación como exploratoria, descriptiva y correlacional, asimismo abarca los enfoques cuantitativo y cualitativo, en donde el marco metodológico se define a partir de la teoría fundamentada para conocer y describir la realidad desde la perspectiva de los agricultores. Además de determinar el tipo de investigación, se define el eje de análisis, el ángulo de la investigación, las preguntas de investigación, las proposiciones, las variables, los indicadores, la hipótesis, el diseño muestral, las herramientas de recolección de datos y su análisis respectivo.

Desarrollo de la investigación. A continuación se describen los pasos del desarrollo de la investigación:

\section{Planteamiento del problema.}

Aunque pareciera que la producción agrícola es una actividad cuyas etapas o proceso están bien estudiados y definidos, en la práctica no lo es tanto pues el hecho de depender de varios factores productivos tierra, capital, mano de obra, tecnología, conocimiento y otros, hace que su estudio y comprensión se torne complejo e interesante. Es precisamente en éstos factores donde se centra la atención de este trabajo pues pretende conocer y describir el grado de interés e importancia que los agricultores atribuyen a los factores productivos, pero en especial al factor tecnológico relacionado con el aprovechamiento de las energias alternas, específicamente la energía solar fotovoltaica, aplicado al bombeo de agua para el riego agrícola. Se da por hecho que las organizaciones agrícolas como tales, tienen como objetivo primario lograr niveles de producción que les permita tener ganancias económicas. Bajo esta perspectiva, se hace el siguiente planteamiento del problema:

"Dado que los agricultores buscan que su producción cumpla con los principios de la producción de 'maximizar la producción y minimizar los costos'; la 'máxima eficacia económica' y/o la 'máxima ganancia', es importante conocer el grado de interés e importancia que los agricultores atribuyen a los factores productivos, y de manera especial al factor tecnológico relacionado con el uso de sistemas fotovoltaicos para el bombeo de agua para el riego agrícola".

Instrumentos de recolección de datos. Primero se determinó realizar una inmersión o acercamiento de tipo exploratorio recurriendo a una entrevista informal no estructurada tipo piloto para dimensionar, entender y comprender lo mejor posible la realidad del agricultor. Después se determinó una muestra piloto para aplicar un cuestionario corto para tener la información necesaria. Posteriormente se elaboró el cuestionario definitivo aplicándolo a la muestra definitiva. Ya con el cuestionario respondido se procedió a clasificar, ordenar, analizar e interpretar los datos para finalmente presentar los resultados y las conclusiones.

Ejes de análisis. Comprende aquellos conceptos del marco teórico que permitieron relacionar heurísticos y acercarnos al hecho social estudiado, en este caso son los tres principios teóricos que estudian la producción agrícola: el principio de los rendimientos decrecientes que estudia la maximización de la producción; el principio de la máxima eficacia económica que estudia la manera de combinar eficazmente los factores productivos fijos y variables en el corto y largo plazo; y el principio de la máxima ganancia que permite determinar la razón ingresos/costos. 
Dimensiones o incrustaciones de análisis. Corresponden a los conceptos denominados ordenadores y que tienen la característica de estar cercanos a la pregunta central y que para este trabajo son los tres principios de la producción, el libre comercio, los apoyos gubernamentales, la tecnología solar fotovoltaica y el riego agrícola.

Ángulo de investigación. Se refiere al heurístico con el cual se realiza un recorte de la totalidad y se acotan aspectos importantes, que para este trabajo son los factores productivos y su impacto en la producción agrícola para maximizar la producción, lograr la máxima eficacia económica y/o lograr la máxima ganancia.

Preguntas centrales y Proposiciones de la investigación. Básicamente se parte de la Pregunta Central, el Ángulo de Investigación y las Dimensiones del Análisis para definir las Preguntas de Investigación y sus Proposiciones.

Figura 1. Proposiciones de la investigación

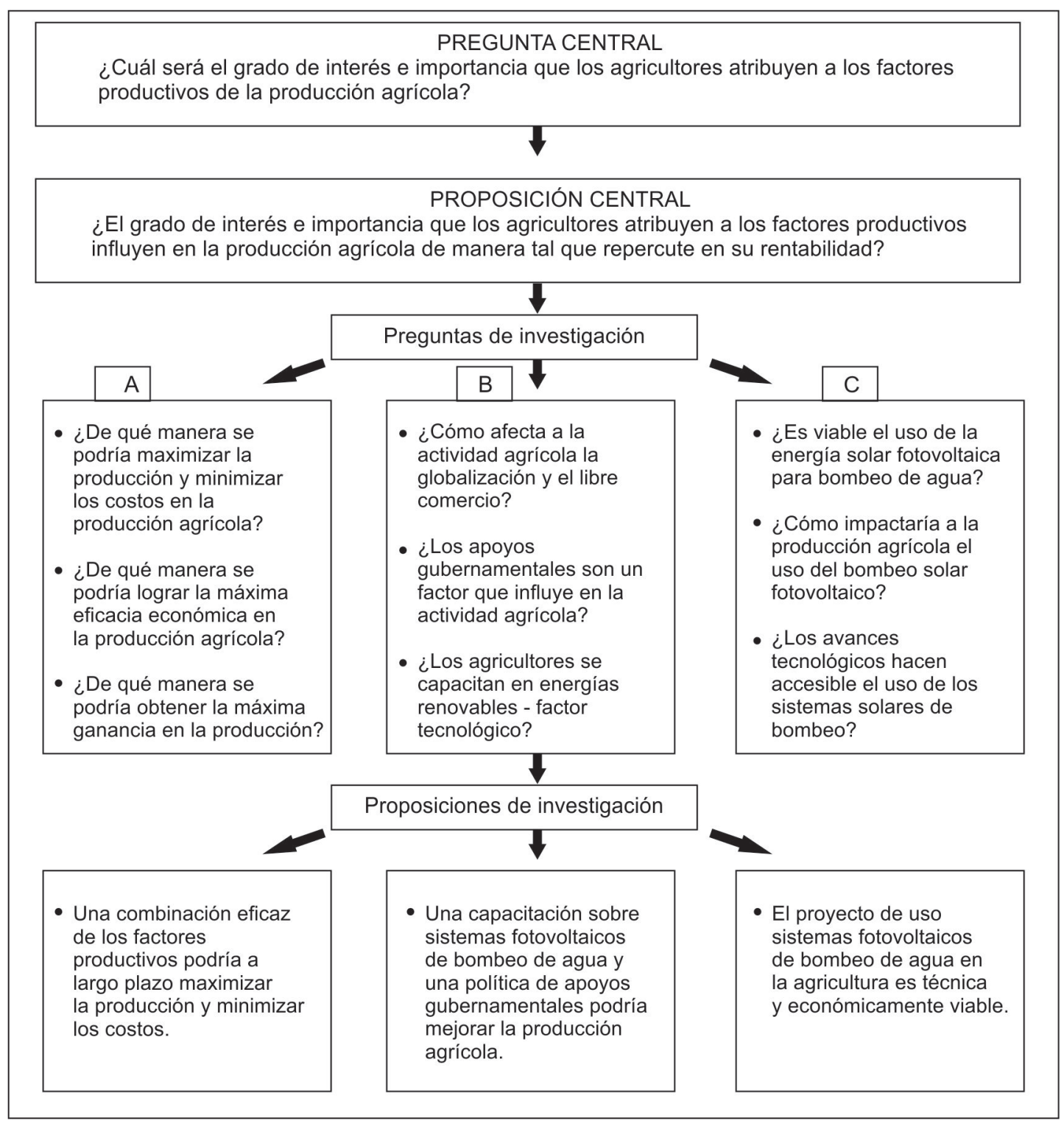


Determinación de variables e indicadores. La importancia de las variables radica en dos atributos, el primero es que sean características observables y el segundo es que sean susceptibles de cambio o variación. La construcción de las variables se hace a partir de las proposiciones de investigación con sus respectivos indicadores.

Figura 2.- Variables e indicadores de la investigación

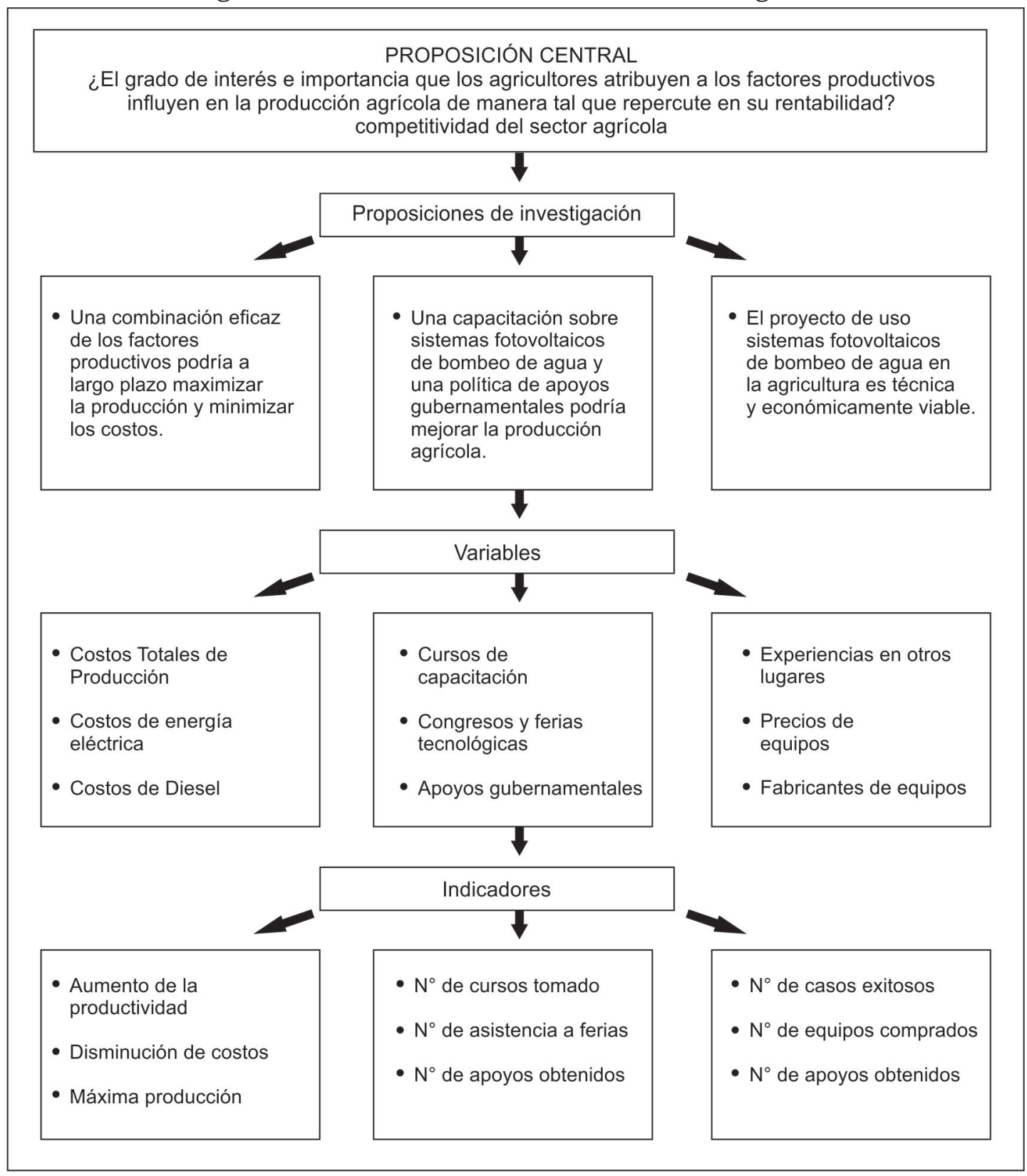




\section{Planteamiento de la hipótesis.}

Partiendo de la pregunta central, las preguntas de la investigación y sus proposiciones respectivas, se formula la hipótesis.

- Hipótesis principal:

o Ho: El grado de interés o nivel de importancia que los agricultores atribuyen al factor productivo tecnológico, es el suficiente para considerar adquirir sistemas fotovoltaicos de bombeo de agua para el riego agrícola para lograr maximizar la producción, minimizar los costos y obtener la máxima eficacia económica.

- Hipótesis alternativa Ha:

o Ha: El grado de interés o nivel de importancia que los agricultores atribuyen al factor productivo de apoyos gubernamentales, es el suficiente para considerar adquirir sistemas fotovoltaicos de bombeo de agua para el riego agrícola para lograr maximizar la producción, minimizar los costos y obtener la máxima eficacia económica.

\section{Diseño muestral.}

Consta de varias etapas:

- Inmersión inicial. - Mediante una entrevista informal no estructurada aplicada a un agricultor elegido al azar.

- Cuestionario piloto. - Aplicado a una muestra piloto pequeña de tamaño $\mathrm{n}=10$ para tener más información para diseñar el cuestionario definitivo.

- Cuestionario definitivo. - Aplicado a una muestra grande de tamaño $\mathrm{n}=92$ con un nivel de confianza del $95.5 \%$ y un error muestral del $3 \%$

- Estratos muestrales. - El marco muestral se dividió en cuatro estratos definidos en función de la ubicación física de los agricultores.

\begin{tabular}{|c|c|c|c|}
\hline Estrato & Población & Fracción & Tamaño de la muestra \\
\hline I & 20 & 0.92 & $\mathrm{n}=19$ \\
\hline II & 32 & 0.92 & $\mathrm{n}_{\text {II }}=30$ \\
\hline III & 15 & 0.92 & $\mathrm{n}_{\text {III }}=14$ \\
\hline IV & 25 & 0.92 & $\mathrm{n}_{\mathrm{IV}}=23$ \\
\hline & $\mathrm{n}=92$ & 0.92 & $\mathrm{n}^{\prime}=86$ \\
\hline
\end{tabular}

Fuente: propia

Validez interna y externa de los datos. Los datos recolectados fueron clasificados en categorías empleando la escala de medición de likert para facilitar su análisis e interpretación. Se adoptó la estrategia de hacer un análisis global y por estratos para tener dos perspectivas de la realidad estudiada. La perspectiva global permitiría de alguna manera garantizar la validez externa, y la perspectiva por estratos además de ofrecer el panorama de cada estrato permitiría garantizar la validez interna al comparar las cuatro sub-muestras o estratos entre sí mediante la herramienta estadística análisis ANOVA para muestras desbalanceadas. 
Revisión, ordenamiento y clasificación de los datos. Los datos recolectados se revisan, ordenan y clasifican, dando inicio a una de las etapas más interesantes de este trabajo que consiste en darle sentido, forma y explicación a los resultados obtenidos, es decir, la culminación del trabajo que permitió darle validez a las proposiciones planteadas, haber establecido la eficacia del proceso planificado, haber verificado las hipótesis según el diseño seleccionado y finalmente haber operacionalizado las variables. En las siguientes tablas se muestran los resultados obtenidos del cuestionario definitivo que permitió procesar 6192 respuestas en total.

\section{Análisis global.}

En este análisis se muestra el grado de interés de los agricultores

\begin{tabular}{|l|c|c|c|c|c|c|c|}
\hline & 1 & 2 & 3 & 4 & 5 & & \\
\hline & Nada & Poco & Regular & Mucho & Demasiado & Total & \\
\hline Capacitación & 249 & 688 & 368 & 217 & 112 & 1634 & $26.4 \%$ \\
\hline & $15.2 \%$ & $42.1 \%$ & $22.5 \%$ & $13.3 \%$ & $6.9 \%$ & & \\
\hline Producción & 135 & 324 & 153 & 76 & 602 & 1290 & $20.8 \%$ \\
\hline & $10.5 \%$ & $25.1 \%$ & $11.9 \%$ & $5.9 \%$ & $46.7 \%$ & & \\
\hline Costos & 80 & 251 & 234 & 256 & 469 & 1290 & $20.8 \%$ \\
\hline Apoyos & $6.2 \%$ & $19.5 \%$ & $18.1 \%$ & $19.8 \%$ & $36.4 \%$ & & \\
\hline & 68 & 174 & 78 & 98 & 442 & 860 & $13.9 \%$ \\
\hline Tecnologías & $7.9 \%$ & $20.2 \%$ & $9.1 \%$ & $11.4 \%$ & $51.4 \%$ & & \\
\hline & $37.2 \%$ & 76 & 0 & 0 & 86 & 258 & $4.2 \%$ \\
\hline $\begin{array}{l}\text { Otra } \\
\text { actividad }\end{array}$ & 285 & 59 & 0 & 11 & 75 & 430 & $6.9 \%$ \\
\hline & $66.3 \%$ & $13.7 \%$ & $0.0 \%$ & $2.6 \%$ & $17.4 \%$ & & \\
\hline Globalización & 51 & 182 & 25 & 65 & 107 & 430 & $6.9 \%$ \\
\hline & $11.9 \%$ & $42.3 \%$ & $5.8 \%$ & $15.1 \%$ & $24.9 \%$ & & \\
\hline Total & 964 & 1754 & 858 & 723 & 1893 & 6192 & $100.0 \%$ \\
\hline
\end{tabular}

Fuente: propia

Considerando ahora a la categoría PRODUCCIÓN como variable dependiente y a las categorías CAPACITACIÓN, COSTOS, APOYOS y TECNOLOGÍAS como variables independientes, se determina la relación o función entre ellas. Las razones de la producción y el resto de las categorias para las escalas "Regular ó Menos" y "Mucho o Más" son las siguientes: 
THE IMPORTANCE OF PRODUCTION FACTORS AND THEIR IMPACT ON THE AGRICULTURAL ORGANIZATIONS IN LEÓN, GUANAJUATO, MEXICO.

Razones de producción y los factores de producción

\begin{tabular}{|l|c|c|c|c|}
\hline \multicolumn{1}{|c|}{ Medida } & Capacitación & Costos & Apoyos & Tecnología \\
\hline Regular o menos & $\mathrm{P}=0.47^{*} \mathrm{FCap}$ & $\mathrm{P}=1.08^{*} \mathrm{FCos}$ & $\mathrm{P}=1.91^{*} \mathrm{FApoy}$ & $\mathrm{P}=1.91^{*} \mathrm{FTec}$ \\
\hline Mucho o más & $\mathrm{P}=2.06^{*} \mathrm{FCap}$ & $\mathrm{P}=0.94^{*} \mathrm{FCos}$ & $\mathrm{P}=1.26^{*} \mathrm{FApoy}$ & $\mathrm{P}=1.26^{*} \mathrm{FTec}$ \\
\hline FUNCIÓN & $\mathrm{P}=0.79^{*} \mathrm{FCap}$ & $\mathrm{P}=1.00^{*} \mathrm{FCos}$ & $\mathrm{P}=1.50^{*} \mathrm{FApoy}$ & $\mathrm{P}=1.50^{*} \mathrm{FTec}$ \\
\hline Ángulo & $\varnothing=38.3^{\circ}$ & $\varnothing=45.0^{\circ}$ & $\varnothing=56.3^{\circ}$ & $\varnothing=56.3^{\circ}$ \\
\hline
\end{tabular}

Fuente: propia

Aquella función cuya pendiente sea mayor tiene la mayor razón de cambio o pendiente sobre la producción agrícola. Los factores productivos con mayor pendiente son la TECNOLOGÍA y los APOYOS GUBERNAMENTALES, seguida de los costos y al final la capacitación. Esto significaría que la producción responde significativamente en mayor proporción a cualquier cambio en los factores tecnología y apoyos gubernamentales, y con menor fuerza a los costos y la capacitación. Sería en esta dirección hacia donde se podrían dirigir los esfuerzos para que el sector agrícola tome un rumbo diferente para que mejoren sus indicadores de productividad, rentabilidad y eficiencia en un mercado globalizado y de libre comercio.

\section{Análisis por estratos.}

Se analizan los estratos para la escala "regular y menos" y se observa que en los cuatro estratos se le asigna MENOR importancia al factor productivo TECNOLOGÍAS ocupando el último lugar. Así mismo se observa que el factor productivo CAPACITACIÓN se le asigna la MAYOR importancia.

\begin{tabular}{|l|c|c|c|c|c|}
\cline { 2 - 6 } \multicolumn{1}{c|}{} & \multicolumn{5}{c|}{ Escala de medición: regular y menos } \\
\hline & Capacitación & Producción & Costos & Apoyos & Tecnologías \\
\hline Estrato I & 288 & 136 & 126 & 71 & 38 \\
\hline Estrato II & 458 & 215 & 199 & 111 & 60 \\
\hline Estrato III & 210 & 98 & 90 & 53 & 28 \\
\hline Estrato IV & 349 & 163 & 150 & 85 & 46 \\
\hline Promedio & 326.3 & 153 & 141.3 & 80 & 43 \\
\hline Desv Std. & 104.6 & 49.2 & 45.7 & 24.5 & 13.5 \\
\hline
\end{tabular}

Fuente: propia 
Para la escala "mucho y más" se observa que en los cuatro estratos se le asigna MENOR importancia al factor productivo TECNOLOGÍA ocupando el último lugar. Asimismo, se observa que el factor productivo COSTOS se le asigna la MAYOR importancia.

\begin{tabular}{|l|c|c|c|c|c|}
\cline { 2 - 6 } \multicolumn{1}{c|}{} & \multicolumn{5}{c|}{ Escala de medición: mucho y más } \\
\hline Estrato I & 159 & 149 & 119 & 73 & 19 \\
\hline Estrato II & 251 & 235 & 189 & 112 & 30 \\
\hline Estrato III & 120 & 112 & 87 & 56 & 14 \\
\hline Estrato IV & 195 & 182 & 145 & 88 & 23 \\
\hline Promedio & 181.3 & 169.5 & 135 & 82.3 & 21.5 \\
\hline Desv Std. & 55.7 & 52.2 & 43.1 & 23.8 & 6.8 \\
\hline
\end{tabular}

Fuente: propia

El factor productivo TECONOLOGÍA ocupa el último lugar en interés en los 4 estratos para ambas escalas "regular y menos" y "mucho y más". Asimismo, el factor productivo CAPACITACIÓN en la escala de "regular y menos" ocupa el primer sitio y el factor productivo COSTOS en la escala de "mucho y más" ocupa la primera posición en nivel de importancia.

\section{Conclusiones.}

La atención se centra en los factores tecnología y apoyos gubernamentales incluidos en la hipótesis principal y alternativa planteadas y se concluye lo siguiente:

- Primera. El factor productivo TECNOLOGÍA ocupa el último lugar en nivel de importancia para los agricultores.

- Segunda. El factor productivo APOYOS GUBERNAMENTALES ocupa el penúltimo lugar en nivel de importancia para los agricultores.

- Tercera. Los factores productivos PRODUCCIÓN y CAPACITACIÓN tienen el mayor interés para los agricultores

- Cuarta. El factor productivo CAPACITACIÓN lo perciben los agricultores desde la perspectiva relacionada con técnicas de riego, manejo de semillas, uso de fertilizantes, pero no en función de cuestiones administrativas, financieras, gerenciales ni mucho menos en energías alternativas.

- Quinta. El planteamiento de la hipótesis principal no se cumple, es decir, no es cierto que el factor tecnológico es determinante en la percepción de los agricultores, sino que ocupa el último lugar en ambas escalas regular y menos y mucho y más. 
- Sexta. El planteamiento de la hipótesis alternativa no se cumple, es decir, no es cierto que el factor apoyos gubernamentales es determinante en la percepción de los agricultores, sino que ocupa el penúltimo lugar en la escala regular y menos y antepenúltimo lugar en la escala mucho y más.

- Séptima. Los agricultores relacionan el factor productivo CAPACITACIÓN solo con aspectos relacionados con la biotecnología y la mejora de cultivos pero no con las energías renovables y mucho menos con el uso de la energía solar fotovoltaica aplicada a la agricultura.

- Octava. Los agricultores relacionan el factor productivo APOYOS GUBERNAMENTALES solo con aspectos relacionados con la compra de maquinaria y equipo como tractores, segadoras, vehículos, sistemas automatizados de riego, pero no en la adquisición de nueva tecnología que use la energía solar fotovoltaica.

- Novena. Se detectó que los agricultores manifiestan un desconocimiento muy importante del factor tecnológico relacionado con las energías alternas aplicadas a la agricultura a pesar de los esfuerzos hechos por las instituciones involucradas incluyendo al gobierno y las instituciones educativas.

- Décima. Se considera como principal aporte de este trabajo el hecho de conocer y describir que la percepción que tienen los agricultores y las políticas de gobierno no están alineadas.

La experiencia obtenida en este trabajo de investigación fue enriquecedora y abre un horizonte amplio lleno de retos, pero con muchas áreas de oportunidad para seguir indagando y descubriendo aspectos relacionados con la producción agrícola y sus factores productivos. Pareciera que el sector agricola solo tiene un camino para sobrevivir y adaptarse a los nuevos tiempos de globalización y libre mercado que exigen altos niveles de competitividad, eficiencia, productividad y rentabilidad, este camino pudiera ser el factor tecnológico a través de las energías renovables aplicadas a la agricultura. Aun cuando las hipótesis principal y alternativa fueron rechazadas, se considera viable y factible asumir que el factor tecnológico pudiera ser el motor que permita que la producción agrícola revierta su actual situación rompiendo paradigmas para lograr la autosuficiencia alimentaria tan necesaria. 


\section{Referencias Bibliográficas.}

Amado , P., \& Blamont, D. (1992). Solar pumping in India, Nepal and Mali -socio-economic feedback of twelve years experiences. 11th EC Photovoltaic Solar Conference. Montreux.

Atkinson, P. (1994). Etnografía. Métodos de investigación. Barcelona: Editorial Paidós.

Farrell, M. (1957). The Measurement of Productive Efficiency. Journal of the Royal Statistical Society, 120(3), 253-290.

Feder, G., Just, R. E., \& Zilberman, D. (1985). Adoption of agricultural innovations in Developing Countries. Washington: The World Bank.

Guidi, D. (1997). A model for the evaluation of sustainable development investments: The case of rural electrification in Moroco, PhD Thesis. Dept. of Agricultural Economics and Policy University of Siena.

Hahn , A., Flores , C., \& Schmidt , R. (1998). Photovoltaic water pumps: Lessons learned from demonstration and field testing projects supported by GTZ. The International Workshop on PV water supply. Marrakech: Morocco.

James, B. (1995). The Impacts of Rural Electrification: Exploring the silence. South Africa: University of Cape Town .

Kittelson, D. A. (1998). Productive uses of electricity -country experiences, prepared for Village Power. Washington D.C.: National Rural Electricity Cooperative Association -NRECA-.

Ramani, K. V. (1992). Rural energy systems in the Asia-Pacific: A survey of their status, planning and management, GTZ/APDC.

Ricketts , C., \& Rawlins, O. (2000). Introducción al Negocio de la Agricultura. Editorial Parainfo Thompson Learning.

Rochin, G., Ortiz, G., \& Ellis, S. (2000). Energía solar para bombeo de agua: Experiencias con ranchos sustentables en Baja California Sur, Fideicomiso de Riesgo Compartido -FIRCO-, La paz, Baja California Sur . Las Cruces, New México: el Instituto de Desarrollo Tecnológico del Suroeste -SWTDI.

Said, S. A. (1989). Case study of solar photovoltaic powered irrigation in the Northern Sudan. Sunworld, 13(2).

Sánchez Crespo, J. L. (1986). Diseño de encuestas por muestreo probabilístico. Madrid España: Instituto Nacional de Estadística.

Sandia Nacional Laboratories. (1998). Guia para el desarrollo de proyectos de bombeo de agua con energía renovable. New México USA: New México State University .

Santos, H., Palacios, V., Exebio , G., \& Chalita, T. (2000). Metodología para evaluar la distribución de costos e ingresos relacionados con el servicio de riego. Revista Agrociencia, 639-649. 
Sapiain, R., Schmidt, R., Flores, C., \& Torres, A. (2000). Evaluación técnica de sistemas de bombeo fotovoltaico. Información tecnológica, 11(6).

Schaefer, J. (1985). Results of a survey of 111 photovoltaic water pumpers in New México. New México: Department of electrical engineering, The citadel, Charleston, S.C. 29409.

Schramm, G. (1993). Rural electrification in LDCs as a tool for economic development: facts and fiction. presented at OPEC Review, winter issue.

Schütz, A. (1932). El problema de la realidad social. Buenos Aires, Argentina: Editorial Amorrortu.

Southwest Technology Development Institute. (s.f.). Guía para el Desarrollo de Proyectos de Bombeo de Agua con Energía Renovable, (Vol. I y II). Las Cruces, New México, U.S.A: New México State University.

Williams, N. (1991). Solar serendipity: photovoltaic rural electrification in Sri Lanka. Solar Today.

Yin, R. K. (1981a). The case study as a serious research strategy, Knowledge: Creation, Diffusion, Utilization. 